University staff mental health literacy, stigma and their experience with students with mental health problems

\author{
Amelia Gulliver $^{1}$ \\ Louise Farrer ${ }^{1}$ \\ Kylie Bennett ${ }^{1}$ \\ Kathleen M Griffiths ${ }^{2}$
}

${ }^{I}$ Centre for Mental Health Research, The Australian National University

${ }^{2}$ Research School of Psychology, The Australian National University

Correspondence concerning this article should address to: Amelia Gulliver, Research Fellow, Centre for Mental Health Research, The Australian National University, Canberra, Australia 2601

T: +61 261259472

E: amelia.gulliver@anu.edu.au

Word count: 3037 


\section{University staff mental health literacy, stigma and their experience of students with mental health problems}

\section{Abstract}

Despite high rates of mental disorders in university students, very few seek professional help. University teaching staff are well placed to connect students with mental health care. However, little is known about university staff attitudes towards and knowledge around mental health problems, or whether these factors influence their experience with and assistance of students with these problems. A total of 224 teaching staff members at the Australian National University, Canberra completed an anonymous online survey via an email link (16.4\% response rate from $n \sim 1370)$. Measures included demographic and professional information, experiences with student mental health, knowledge of depression (literacy), and attitudes towards depression (stigma). Level of personal stigma did not predict whether a teaching staff member would approach a student to assist with mental health problems. Teaching staff with higher levels of depression literacy $(\mathrm{OR}=1.14, p=.007)$ were more likely to feel sufficiently informed to help students with mental health problems. Ensuring staff complete mental health literacy training and have adequate skills to respond appropriately to students with mental health problems may help in connecting young people to appropriate care in a university context.

\section{Keywords}

university, teaching staff, mental health, stigma, literacy 
The prevalence of mental disorders is greatest among young adults aged 16-24 years (Australian Bureau of Statistics, 2007), an age at which many young people are enrolled in higher education (Birrell and Edwards 2007). Despite this, research indicates that very few young people seek help for mental health problems (Rickwood and Thomas 2012). As potential gatekeepers (Frederico and Davis 1996), university teaching staff members are well positioned to offer an initial point of contact for referral to appropriate sources of professional help. However, whilst previous research has investigated mental health knowledge and stigma in secondary school teachers (Jorm, Kitchener et al. 2010, Kutcher, Wei et al. 2013), little is known about the attitudes of university teaching staff towards students with mental health problems (stigma), about staff levels of mental health literacy, or whether these factors impact on their capacity or willingness to assist students with mental health problems.

In addition to the age-related association (Australian Bureau of Statistics, 2007), issues specific to university life including academic and social expectations during the transition to university can also put students at risk of mental health problems (Cleary, Walter et al. 2011). Mental disorders are responsible for a high proportion of disability burden in university students (Mowbray, Megivern et al. 2006, Hunt and Eisenberg 2010, Buchanan 2012) and poor mental health can also have a negative impact on academic performance and participation (Eisenberg, Gollust et al. 2007, Reavley and Jorm 2010, Buchanan 2012). Despite these consequences, less than one-third seek help for mental health problems (Essau 2005, Slade, Johnston et al. 2009). Common mental disorders in young people such as depression and anxiety disorders are treatable, and potentially preventable (Barrera, Torres et al. 2007, Bienvenu and Ginsburg 2007, Waddell, Hua et al. 2007). Appropriate help-seeking is therefore particularly important for the early detection and treatment of mental disorders, and recovery (Dawson, Grant et al. 2006, Yung, Killackey et al. 2007). 
Very few students disclose a mental health problem at university. It is possible students may fear being stigmatised by self-identifying their mental illness. Perceived stigma [a perception that others hold stigmatising views (Griffiths, Christensen et al. 2004)], may play a role in low levels of disclosure and help-seeking. Students with higher levels of perceived stigma from family and friends have been found to be significantly less likely to seek help from university counselling services (D'Amico, Mechling et al. 2016) and very few ( $0.7 \%$ of 2.5 million) disclosed their mental health condition to the university, a percentage well under prevalence estimates (Equality Challenge Unit 2012). Students have reported fearing being labelled with a mental health condition or being discontinued from their course (Freeburn and Sinclair 2009) by the university should they disclose their mental health status. It is likely that student concerns about the potential impact of disclosure on their university career reflects students' more general levels of perceived stigma of mental health conditions which may extend to their perception of the views of university teaching staff.

In the educational environment, teaching staff have been identified as potential gatekeepers who are able to help connect distressed individuals with mental health services (Frederico and Davis 1996). Accordingly, teaching staff at universities such as lecturers and tutors are often approached by students with mental health problems (Leane and Shute 1998). Therefore, university teaching staff members may be important in linking students with professional help. However, many teachers may not feel sufficiently equipped to assist students with these problems (Rickwood, Deane et al. 2005, Huyton 2009) and may lack knowledge about mental disorders (Walter, Gouze et al. 2006). In addition, they may hold attitudes that actively impede their willingness to facilitate access to professional sources of help for the young person (Wilson and Deane 2000). Previous research has measured stigma and literacy in secondary school teachers (Jorm, Kitchener et al. 2010, Kutcher, Wei et al. 2013), non-academic university staff members [i.e., student affairs staff (Massey, Brooks et 
al. 2010)], or examined combined data from both teaching and professional university staff (Wynaden, McAllister et al. 2014). Thus, there is a paucity of research on stigmatising attitudes towards mental disorders as well as the mental health literacy of university teaching staff. The current study aims to address this gap in the research by investigating university teaching staff literacy and stigma around a common mental health problem (depression), and assessing the influence of these factors on their experience with and assistance of students with mental health problems.

\section{Method}

\section{Participants}

Participants were 224 academic staff from The Australian National University (ANU), a moderate-sized university comprising 15,821 students and approximately 1370 teaching staff in Canberra, Australia. The sample and method has been described in detail previously (Farrer, Gulliver et al. 2015). Table 1 presents the demographic and professional characteristics of the participants. The participants on average were 41.6 years of age $(S D=$ 12.45) and comprised 113 men, and 110 women (other, $n=1$ ), with the majority Caucasian or from European descent (76.2\%). Most were lecturers (61.2\%) or supervisors of higher degree research students (52.2\%), and the average duration of teaching experience at university level was approximately 10 years. 


\section{Procedure}

An email was sent to academic staff and postgraduate students (including casual tutors who may not be on the academic staff email list) inviting those with teaching responsibilities to complete an anonymous online survey during November and December 2013. A full list of teaching staff was not available from the university; thus the estimated population sample was 1370 academic teaching staff members based on university records assessed at the time of the survey distribution. Staff/post graduate scholars were eligible to complete the online questionnaire if they indicated that they were involved in teaching and/or supervision of undergraduate or postgraduate students (e.g., as a lecturer, tutor, course convenor, and/or research supervisor). Staff provided informed consent online by selecting a box marked "Yes" indicating that they agreed that they had read and understood the information on study's privacy and consent page. Ethics approval for the study was granted by the ANU Human Research Ethics Committee (2013/449).

\section{Measures}

\section{Demographic and professional characteristics}

Demographic and professional information collected included age, gender, ethnicity, teaching role (i.e., lecturer, tutor, course convenor, research supervisor) and level (e.g., first year, honours, postgraduate etc.), academic discipline, and number of years teaching or supervising at the university level.

Previous experience with student mental health.

Previous experience assisting students with mental health problems: A series of questions developed by the researchers and adapted from Reinke et al. (2011) to assess participants' experiences with providing assistance to students with mental health problems. Participants 
were asked if they a) feel sufficiently informed to respond appropriately to a student with a mental health problem, and whether they have ever (b) taught a student with a mental health problem, (c) initiated a conversation with a student about their mental health problem(s), (d) been approached or confided in by a student about their mental health problem(s), (e) been told by a student that they were having suicidal thoughts.

\section{Mental health literacy}

Mental health literacy specific to depression was assessed using the Depression Literacy questionnaire [D-Lit, (Griffiths, Christensen et al. 2004)]. This measure comprises 22 items each assessing the participants' knowledge about depression. Respondents are required to indicate whether each statement is "true", "false", or "I don't know". Examples include "Loss of confidence and poor self-esteem may be a symptom of depression" (true) and "Depression does not affect your memory and concentration" (false). One point is scored for each correct answer. Total scores range from 0-22 with higher scores indicating higher knowledge levels. Cronbach's Alpha for the D-Lit in the current study was $0.83(n=201)$. Previous studies using the D-Lit reported very good internal consistency $(\alpha=0.70-0.92$ respectively; (Kiropoulos, Griffiths et al. 2011, Gulliver, Griffiths et al. 2012)), as well as adequate validity, and very good test-retest reliability $[r=0.71-0.91$; (Kiropoulos, Griffiths et al. 2011, Gulliver, Griffiths et al. 2012)].

\section{Depression Stigma}

Stigma towards depression was assessed using the Personal Stigma subscale of the Depression Stigma Scale [DSS (Griffiths, Christensen et al. 2004)]. This scale comprises 9 items measuring the participants' own stigmatising attitudes towards depression. Each item consists of a statement such as "Depression is not a real medical illness" or "if I had 
depression I would not tell anyone”. Each statement is scored on a 5-point Likert-type scale ranging from 0 (strongly disagree) to 4 (strongly agree). Total scores ranges from 0-36 with higher scores indicating higher personal stigmatising attitudes towards depression.

Cronbach's Alpha for the current study was $0.84(n=203)$. Previous research has reported adequate internal consistency for the DSS in studies of adults $[\alpha=0.77$ (Griffiths, Christensen et al. 2008, Gulliver, Griffiths et al. 2012)], as well as acceptable validity and test-retest reliability [( $r=0.66-0.86)$ (Griffiths, Christensen et al. 2004, Gulliver, Griffiths et al. 2012)].

\section{Analyses}

Quantitative data analyses were conducted using SPSS Version 23 (IBM Corp, 2014). Binary logistic regression analyses were used to examine the relationship between participant demographic characteristics, depression stigma, and literacy, and participants' previous experience with student mental health problems. Separate analyses were undertaken for each of the five previous experience items (dependent variables); independent variables were demographic status were: age (years); gender $($ male $=0$, female $=1)$; teaching experience at a university level (years); teaching role (course convenor, lecturer, tutor, or research supervisor, each coded as yes $=1$, no $=0)$; discipline taught $(0=$ medicine, health and behavioural sciences, 1 = Other: physical, mathematical and computer sciences, economics and engineering, arts, social sciences and law); and the participants' depression stigma and literacy scores. In addition, separate binary logistic regression analyses were undertaken to examine the above potential predictors (excluding stigma) of depression stigma and depression literacy. The independent variables were entered simultaneously into each model. All dependent variables were binary coded $($ yes $=1$, no $=0)$. 


\section{Results}

\section{Response rate}

A response rate of $16.4 \%(224 / 1370)$ was achieved based on the estimated staff numbers provided by the university at the point of survey distribution.

\section{Stigma}

The mean depression stigma score for the participants was $7.23(n=203, S D=5.07$, range $=$ $0-36)$. The regression analysis showed that those with higher depression literacy $(t=-4.85, p$ $<.001)$ had significantly lower levels of stigma than those with lower levels of literacy. No other characteristics were significantly predictive of stigma scores.

\section{Mental health literacy}

The mean depression literacy score for the participants was $13.78(n=201, S D=4.28$, range $0-22)$. The regression model demonstrated that female teaching staff $(t=3.08, p=.002)$, and those from a health/behavioural science background $(t=-5.65, p<.001)$ had significantly higher levels of depression literacy than those from other disciplines. No other variables were significant.

\section{Experiences with student mental health}

Table 1 presents the staff experience of teaching students with mental health problems. The five regression models examining experience with student mental health showed that teaching staff with more years of experience were more likely to have previously taught a student who appeared to be experiencing a mental health problem $(\mathrm{OR}=1.11, \mathrm{~S} . \mathrm{E} .=.042, p$ $=.011)$, those with higher depression literacy were more likely to have initiated a 
conversation with a student to discuss their mental health $(\mathrm{OR}=1.13$, S.E. $=.051, p=.016)$. Course convenors $(\mathrm{OR}=3.76, \mathrm{~S} . \mathrm{E} .=.457, p=.004)$ and those with higher depression literacy $(\mathrm{OR}=1.14, \mathrm{~S} . \mathrm{E} .=.049, p=.008)$ were more likely to have been approached by a student to discuss their mental health. In addition, higher level of depression literacy was associated with feeling sufficiently informed to respond appropriately to a student with a mental health problem $(\mathrm{OR}=1.14$, S.E. $=.048, p=.007)$. Level of personal stigma did not significantly predict any of the outcome variables including whether a teaching staff member would approach a student to assist with mental health problems. Finally, no demographic or personal characteristics were associated with a staff member reporting that had student had told them about suicidal thoughts.

\section{Discussion}

Overall this study found that staff with higher levels of depression literacy were more likely to engage with students with mental health problems. However, higher levels of stigmatising attitudes towards depression did not independently predict whether a teaching staff member would approach a student to assist with a mental health problem.

Staff in the current survey had high levels of depression literacy. The mean depression literacy score for the D-Lit measure $(13.78, S D=4.28)$ appears higher than figures reported in a previous study of Greek and Italian-born Australian immigrants $(8.17-10.61, S D=3.28$ $4.29 n=202$ ), yet comparable to a group of community adults experiencing symptoms of depression [mean range $=12.9-13.2, S D=3.1-3.6, n=525 ;$ (Griffiths, Christensen et al. 2004)] and Australian elite athletes [12.05, $S D=3.22, n=40$; (Gulliver, Griffiths et al. 2012)]. Women and those from health and behavioural science disciplines were more likely to demonstrate higher depression literacy than men in the current sample, which supports previous studies examining the effect of gender (Swami 2012) and discipline (Laws and 
Fiedler 2012) on mental health literacy. Given that staff with higher depression literacy are more likely to assist students with mental health problems, these findings suggest that there may be value in introducing interventions designed to increase mental health literacy, particularly in males and disciplines where mental health literacy is lower. It is also important to note that course convenors were also significantly more likely to be approached by a student with a mental health problem. Therefore, also ensuring that these staff members have adequate knowledge to assist students is critical.

The absence of an independent effect of stigma in this study may reflect, at least in part, the lower levels of stigma in this sample. On average university teaching staff disagreed with stigmatising statements about people with depression. Which, given teaching staff are a highly educated group, is consistent with previous research showing that higher levels of stigma were associated with lower levels of education (Griffiths, Christensen et al. 2008). The level of personal stigma in the current study participants $(M=7.23, S D=5.07)$ was low compared with mean personal stigma scores in other community samples, including a US study of university staff and graduate students $(M=12.70, S D=7.2, n=42)$ (Finkelstein and Lapshin 2007), a sample of Dutch community-based adults $(M=12.00, S D=4.34, n=253)$ (Boerema, Zoonen et al. 2016), a study of Australian adults with elevated depression symptoms $(M=9.7-10.7, S D=5.0, n=525)($ Griffiths, Christensen et al. 2004) and a study of Greek and Italian immigrants living in Australia $(M=18.38-18.44, S D=4.66-4.78, n=$ 202) (Kiropoulos, Griffiths et al. 2011). Jorm et al.(Jorm, Kitchener et al. 2010) reported that only one-third of high school teachers $(n=321)$ strongly disagreed that people with depression could "snap out of it", whereas in the current study, double the proportion of staff $(64.5 \% ; n=203)$ strongly disagreed with this statement, indicating lower stigma in the current sample. However, importantly, stigma did not impact on a staff member willingness to approach a student to assist with mental health problems. This is a key finding as it 
indicates that staff may be unlikely to allow personal beliefs interfere with their professional judgement, as previous research has suggested (Wilson and Deane 2000). It is possible that practical considerations for teaching staff are highly important, including that other influences such as staff performance and student outcomes may important motivators despite personal attitudes.

Teaching staff with higher depression literacy were more likely to be approached by a student, independent of their gender or teaching field. It is possible that students are more likely to approach those who they perceive to have a better understanding of mental health issues. An alternative explanation is that teaching staff with a higher level of depression literacy may due to this awareness, respond in a way which does not discourage the student from initiating the intended conversation about their mental health status. Moreover, it is possible that students may share experiences with their peers and this may encourage other students to approach a staff member in particular. Indeed, staff who had higher depression literacy also tended to have less stigmatising attitudes towards depression. This is consistent with several meta-analyses of the outcomes of programs for reducing the stigma associated with mental health problems which demonstrate that educational interventions including literacy are effective at reducing personal stigma (Corrigan, Morris et al. 2012, Griffiths, Carron-Arthur et al. 2014), and specifically for depression (Griffiths, Carron-Arthur et al. 2014).

\section{Limitations}

There are several limitations to this study. These include that the university teaching staff members self-selected to participate in the current study. Thus, those with a particular interest or experience in student mental health may have completed the survey, and the sample may not be representative of all teaching staff. This might explain in part the low levels of stigma 
and high levels of depression literacy. Further, we were not able to calculate an accurate rate of participation given the lack of records available from the university. In addition, the sample comprised a single university, and thus may not be representative of all university staff. Finally, only attitudes and knowledge around one common mental disorder were assessed (depression) so it is possible that staff may have had more knowledge or stigmatising attitudes towards other mental health problems.

\section{Conclusions}

This study showed that university staff attitudes towards depression did not influence whether they would approach a student to assist with mental health problems. The study found that in general, university staff appeared to have relatively high literacy and low perceptions of stigma around depression. Those who had higher depression literacy were more likely to feel sufficiently informed to help a student with a mental health problem. However, whilst this research has provided valuable information around staff literacy and attitudes, further work is required to determine the actions academics engage in when confronted with mental health issues, their personal confidence in handling these issues, and how they learn from these experiences. Given their gatekeeper role, ensuring staff complete training to ensure adequate mental health literacy and feel equipped to respond appropriately is critical to connecting young people to mental health care in a university teaching context.

\section{Acknowledgements}

The authors wish to acknowledge Jade Chan for assistance with developing the survey questions. 


\section{Disclosure}

The Authors declare that there is no conflict of interest

\section{Funding}

This work was supported by the Young and Well CRC (youngandwellcrc.org.au). The Young and Well CRC is established under the Australian Government's Cooperative Research Centres Program. KMG was supported by a National Health and Medical Research Council Fellowship (No.1059620).

\section{References}

(2007). Australian Bureau of Statistics: National Survey of Mental Health and Wellbeing: Summary of Results.

Barrera, A. Z., L. D. Torres and R. F. Munoz (2007). "Prevention of depression: the state of the science at the beginning of the 21 st Century." Int Rev Psychiatry 19(6): 655-670.

Bienvenu, O. J. and G. S. Ginsburg (2007). "Prevention of anxiety disorders." Int Rev Psychiatry 19(6): 647-654.

Birrell, B. and D. Edwards (2007). "Half of Australian youth aged 18-20 are not in training." University World News(November).

Boerema, A. M., K. Zoonen, P. Cuijpers, C. J. Holtmaat, L. B. Mokkink, K. M. Griffiths and A. M. Kleiboer (2016). "Psychometric Properties of the Dutch Depression Stigma Scale (DSS) and Associations with Personal and Perceived Stigma in a Depressed and Community Sample." PLoS One 11(8): e0160740.

Buchanan, J. L. (2012). "Prevention of depression in the college student population: a review of the literature." Arch Psychiatr Nurs 26(1): 21-42. 
Cleary, M., G. Walter and D. Jackson (2011). "'Not always smooth sailing": mental health issues associated with the transition from high school to college." Issues Ment Health Nurs 32(4): 250-254.

Corrigan, P. W., S. B. Morris, P. J. Michaels, J. D. Rafacz and N. Rusch (2012).

"Challenging the public stigma of mental illness: a meta-analysis of outcome studies." Psychiatr Serv 63(10): 963-973.

D'Amico, N., B. Mechling, J. Kemppainen, N. R. Ahern and J. Lee (2016). "American College Students' Views of Depression and Utilization of On-Campus Counseling Services." J Am Psychiatr Nurses Assoc 22(4): 302-311.

Dawson, D. A., B. F. Grant, F. S. Stinson and P. S. Chou (2006). "Estimating the effect of help-seeking on achieving recovery from alcohol dependence." Addiction 101(6): 824-834.

Eisenberg, D., S. E. Gollust, E. Golberstein and J. L. Hefner (2007). "Prevalence and correlates of depression, anxiety, and suicidality among university students." Am J Orthopsychiatry 77(4): 534-542.

Equality Challenge Unit (2012). Equality in higher education: statistical report 2012 Part 2: students. London, Equality Challenge Unit.

Essau, C. A. (2005). "Frequency and patterns of mental health services utilization among adolescents with anxiety and depressive disorders." Depress Anxiety 22(3): 130-137.

Farrer, L., A. Gulliver, K. Bennett and K. M. Griffiths (2015). "Exploring the acceptability of online mental health interventions among university teaching staff: Implications for intervention dissemination and uptake." Internet Interventions 2(3): 359-365.

Finkelstein, J. and O. Lapshin (2007). "Reducing depression stigma using a web-based program." Int J Med Inform 76(10): 726-734. 
Frederico, M. and C. Davis (1996). Gatekeeper training and youth suicide prevention. Report for Youth Suicide Prevention Initiative: Education and Training Consultancy. D. o. H. a. F. Services. Canberra.

Freeburn, M. and M. Sinclair (2009). "Mental health nursing students' experience of stress: burdened by a heavy load." J Psychiatr Ment Health Nurs 16(4): 335-342.

Griffiths, K. M., B. Carron-Arthur, A. Parsons and R. Reid (2014). "Effectiveness of programs for reducing the stigma associated with mental disorders. A meta-analysis of randomized controlled trials." World Psychiatry 13(2): 161-175.

Griffiths, K. M., H. Christensen and A. F. Jorm (2008). "Predictors of depression stigma." BMC Psychiatry 8: 25.

Griffiths, K. M., H. Christensen, A. F. Jorm, K. Evans and C. Groves (2004). "Effect of webbased depression literacy and cognitive-behavioural therapy interventions on stigmatising attitudes to depression: randomised controlled trial." British Journal of Psychiatry 185: 342-349.

Gulliver, A., K. M. Griffiths, H. Christensen, A. Mackinnon, A. L. Calear, A. Parsons, K. Bennett, P. J. Batterham and R. Stanimirovic (2012). "Internet-based interventions to promote mental health help-seeking in elite athletes: an exploratory randomized controlled trial." Journal of medical Internet research 14(3): e69.

Hunt, J. and D. Eisenberg (2010). "Mental health problems and help-seeking behavior among college students." J Adolesc Health 46(1): 3-10.

Huyton, J. (2009). "Significant Personal Disclosure: exploring the support and development needs of HE tutors engaged in the emotion work associated with supporting students." Journal of Learning Development in Higher Education(1). 
Jorm, A. F., B. A. Kitchener, M. G. Sawyer, H. Scales and S. Cvetkovski (2010). "Mental health first aid training for high school teachers: a cluster randomized trial." BMC Psychiatry 10: 51.

Kiropoulos, L. A., K. M. Griffiths and G. Blashki (2011). "Effects of a multilingual information website intervention on the levels of depression literacy and depressionrelated stigma in Greek-born and Italian-born immigrants living in Australia: a randomized controlled trial." J Med Internet Res 13(2): e34.

Kutcher, S., Y. Wei, A. McLuckie and Bullock (2013). "Educator mental health literacy: a programme evaluation of the teacher training education on the mental health \& high school curriculum guide." Advances in School Mental Health Promotion 6(2): 83-93.

Laws, T. A. and B. A. Fiedler (2012). "Universities' expectations of pastoral care: trends, stressors, resource gaps and support needs for teaching staff." Nurse Education Today 32(7): 796-802.

Leane, W. and R. Shute (1998). "Youth suicide: the knowledge and attitudes of Australian teachers and clergy." Suicide Life Threat Behav 28(2): 165-173.

Massey, J., M. Brooks, J. Burrow and C. Sutherland (2010). Increasing mental health literacy among student affairs staff: assessing 'mental health first aid'. Kingston, Canada, Queen's University.

Mowbray, C. T., D. Megivern, J. M. Mandiberg, S. Strauss, C. H. Stein, K. Collins, S. Kopels, C. Curlin and R. Lett (2006). "Campus mental health services: recommendations for change." Am J Orthopsychiatry 76(2): 226-237.

Reavley, N. and A. F. Jorm (2010). "Prevention and early intervention to improve mental health in higher education students: a review." Early Interv Psychiatry 4(2): 132-142. 
Reinke, W. M., M. Stormont, K. C. Herman, R. Puri and N. Goel (2011). "Supporting Children's Mental Health in Schools: Teacher Perceptions of Needs, Roles, and Barriers." School Psychology Quarterly 26(1): 1-13.

Rickwood, D., F. Deane, C. Wilson and J. Ciarrochi (2005). "Young people's help-seeking for mental health problems." AeJAMH (Australian e-Journal for the Advancement of $\underline{\text { Mental Health) }}$ 4(3).

Rickwood, D. and K. Thomas (2012). "Conceptual measurement framework for help-seeking for mental health problems." Psychol Res Behav Manag 5: 173-183.

Slade, T., A. Johnston, M. Teesson, H. Whiteford, P. Burgess, P. Pirkis and S. Saw (2009). The Mental health of Australians 2: report on the 2007 national survey of mental health and wellbeing. D. o. H. a. Ageing. Canberra, Department of Health and Ageing.

Swami, V. (2012). "Mental health literacy of depression: gender differences and attitudinal antecedents in a representative British sample." PLoS One 7(11): e49779.

Waddell, C., J. M. Hua, O. M. Garland, R. D. Peters and K. McEwan (2007). "Preventing mental disorders in children: a systematic review to inform policy-making." $\underline{\text { Can J }}$ Public Health 98(3): 166-173.

Walter, H. J., K. Gouze and K. G. Lim (2006). "Teachers' beliefs about mental health needs in inner city elementary schools." J Am Acad Child Adolesc Psychiatry 45(1): 61-68.

Wilson, C. and F. Deane (2000). If we can't seek help, how can the kids? Illawarra Institute for Mental Health Conference. Wollongong.

Wynaden, D., M. McAllister, J. Tohotoa, O. Al Omari, K. Heslop, R. Duggan, S. Murray, B. Happell and L. Byrne (2014). "The silence of mental health issues within university environments: a quantitative study." Arch Psychiatr Nurs 28(5): 339-344. 
Yung, A. R., E. Killackey, S. E. Hetrick, A. G. Parker, F. Schultze-Lutter, J. Klosterkoetter, R. Purcell and P. D. McGorry (2007). "The prevention of schizophrenia." Int Rev Psychiatry 19(6): 633-646. 
Table 1. Demographic and professional characteristics of the sample $(n=224)$

\section{Characteristic}

\begin{tabular}{lr}
\hline Age $(M, S D)$ & $41.6(12.5)$ \\
Gender $(n, \%)$ & \\
Male & $113(50.4)$ \\
Female & $110(49.1)$ \\
Other & $1(0.4)$
\end{tabular}

Teaching role $(n, \%)$

Lecturer

$137(61.2)$

Higher degree ( $\mathrm{PhD}$, Masters) research supervisor

$117(52.2)$

Tutor

$96(42.9)$

Course convenor

$82(36.6)$

Ethnicity ( $n, \%)$

Caucasian/European

$170(76.2)$

Asian/Indian

$40(17.9)$

Latino/South American

$6(2.7)$

Multiple/Other

$5(2.2)$

Aboriginal/Torres Strait Islander

$2(0.9)$

\section{Discipline}

Arts and Social Sciences

$67(30.2)$

Medicine, Biological and Health Sciences

$51(23.0)$

Physical and Mathematical Sciences

$47(21.2)$

Engineering and Computer Science

$21(9.5)$

Business and Economics

$19(8.6)$

Law

$15(6.8)$ 
Multidisciplinary

Teaching/supervising level

Higher undergraduate students (second, third, fourth year)

$152(67.9)$

Postgraduate research students

$110(49.1)$

Honours students

$94(42.0)$

Postgraduate coursework students

$91(40.6)$

First year undergraduate students

$84(37.5)$

Other

Years teaching/supervising at university level $(M, S D)$

$10.8(9.6)$

\section{Experience with student mental health}

Have previously taught a student with a mental health problem $(n, \%) \quad 146(65.2)$

Have been approached by a student to discuss their mental health $(n, \%) \quad 125(55.8)$

Have initiated a conversation with a student about the student's mental

health $(n, \%)$

Have been told by a student the student was experiencing suicidal

thoughts $(n, \%)$

Feel sufficiently informed to respond appropriately to a student with a

mental health problem $(n, \%)$ 